\title{
Jane Jacobs en Barcelona: las condiciones para la vitalidad urbana y su relación con la movilidad cotidiana
}

\author{
Xavier Delclòs-Alió \\ Carme Miralles-Guasch \\ Universitat Autònoma de Barcelona. Departament de Geografia \\ xavier.delclos@uab.cat \\ carme.miralles@uab.cat
}

\begin{abstract}
Resumen
Este estudio evalúa las ideas de Jane Jacobs, conocida activista y pensadora urbana, aplicadas a la ciudad de Barcelona. Para ello, en primer lugar, se sintetizan las seis condiciones básicas de Jacobs para la vitalidad urbana por distritos y por barrios. En segundo lugar, se relaciona la vitalidad urbana en los barrios con los principales patrones de movilidad cotidiana de las personas residentes. Si bien los barrios de Barcelona presentan considerables diferencias por lo que se refiere a sus condiciones para la vitalidad, se ha detectado a su vez una clara relación de dichos niveles con las pautas de movilidad cotidiana de la población, con un especial vínculo con los desplazamientos a pie y de proximidad. Este trabajo muestra de qué modo la aplicación de las ideas de Jacobs a una ciudad mediterránea como Barcelona permite analizar los efectos que generan distintos tipos de tejidos urbanos.
\end{abstract}

Palabras clave: Jane Jacobs; vitalidad urbana; movilidad cotidiana; Barcelona

Resum. Jane Jacobs a Barcelona: les condicions per a la vitalitat urbana i la seva relació amb la mobilitat quotidiana

Aquest estudi avalua les idees de Jane Jacobs, coneguda activista i pensadora urbana, aplicades a la ciutat de Barcelona. Per fer-ho, en primer lloc, se sintetitzen les sis condicions bàsiques de Jacobs per a la vitalitat urbana per districtes i per barris. En segon lloc, es relaciona la vitalitat urbana en el barri amb els principals patrons de mobilitat quotidiana de les persones residents. Si bé els barris de Barcelona presenten considerables diferències pel que fa a les seves condicions per a la vitalitat, al seu torn s'ha detectat una clara relació d'aquests nivells amb les pautes de mobilitat quotidiana de la població, amb un especial vincle amb els desplaçaments a peu i de proximitat. Aquest treball mostra de quina manera l'aplicació de les idees de Jacobs a una ciutat mediterrània com Barcelona permet analitzar els efectes que generen diferents tipus de teixits urbans.

Paraules clau: Jane Jacobs; vitalitat urbana; mobilitat quotidiana; Barcelona 
Résumé. Jane Jacobs à Barcelone : les conditions de la vitalité urbaine et leur rapport avec la mobilité quotidienne

Cette étude évalue les idées de Jane Jacobs, militante et penseuse urbaine reconnue, à travers son application à la ville de Barcelone. Pour cela, tout d'abord, les six conditions de base de Jacobs pour la vitalité urbaine au niveau des districts et des quartiers sont synthétisées. Ensuite, la vitalité urbaine au niveau des quartiers est associée aux principales tendances de la mobilité quotidienne de leurs résidents. Bien que les quartiers de Barcelone présentent des différences considérables en termes de conditions de vitalité, une relation claire entre ces niveaux et les schémas de mobilité quotidienne de leurs résidents a été détectée, avec un lien plus particulier concernant la mobilité à pied et de proximité. Ce travail montre comment l'application des idées de Jacobs à une ville méditerranéenne telle que Barcelone permet d'analyser les effets générés par différents types de tissus urbains.

Mots-clés: Jane Jacobs; vitalité urbaine; mobilité quotidienne; Barcelone

Abstract. Jane Jacobs in Barcelona: Conditions for urban vitality and their relationship with everyday mobility

This study evaluates the ideas of the well-known activist and urban thinker Jane Jacobs through their application to the city of Barcelona. To this end, Jacobs' six basic conditions for urban vitality are first synthesized at the district and neighborhood levels. Secondly, urban vitality at the neighborhood level is related to the main patterns of daily mobility of its residents. Although neighborhoods in Barcelona vary considerably in terms of their vitality conditions, a clear relationship has been identified between these vitality levels and residents' daily mobility patterns, particularly with regard to walking mobility and proximity. This work shows how the application of Jacobs' ideas to a Mediterranean city such as Barcelona allows analyzing the effects caused by different types of urban fabrics.

Keywords: Jane Jacobs; urban vitality; everyday mobility; Barcelona

\section{Sumario}

$\begin{aligned} \text { 1. Introducción } & \text { 4. Resultados } \\ \text { 2. Condiciones para la vitalidad urbana y } & \text { 5. Conclusión } \\ \text { el «ballet de la acera» } & \text { Referencias bibliográficas } \\ \text { 3. Metodología } & \end{aligned}$

\section{Introducción}

Jane Jacobs, activista y teórica urbana estadounidense, es una de las figuras más influyentes en la historia del estudio y la comprensión de la ciudad ${ }^{1}$. En su famosa obra Muerte y vida de las grandes ciudades (1961), Jacobs reflexionó acerca de las características que hacían de las ciudades, y especialmente de sus

1. Según la revista electrónica Planetizen, en 2009 Jane Jacobs fue elegida como la pensadora urbana más influyente de la historia (<https://www.planetizen.com/topthinkers>, fecha de la última consulta: 05/07/2018). 
barrios, entornos llenos de vida o, por el contrario, que resultaran espacios urbanos inertes. Su propia experiencia cotidiana y las observaciones que realizó meticulosamente en Greenwich Village (Nueva York), donde residió gran parte de su vida, fueron su base metodológica. De este modo, Jane Jacobs aportó al urbanismo una visión teórica sobre los atributos que el entorno construido debería presentar para promover y garantizar calles repletas de gente, entendidas como uno de los reflejos más claros de la vitalidad urbana (Sung et al., 2013).

Sus ideas han sido redescubiertas y revisitadas de forma recurrente tanto en la esfera de la investigación como de la planificación urbana, especialmente en los últimos años (Hirt y Zahm, 2012; Schubert, 2014). Concretamente, en el ámbito académico son cada vez más numerosos los estudios que tratan de llevar al terreno aplicado las ideas teóricas de Jacobs, con el uso de fuentes y métodos actuales (Delclòs-Alió y Miralles-Guasch, 2018; Sulis et al., 2018). La influencia de Jane Jacobs ha sido especialmente relevante en el contexto anglosajón, y se ha considerado que su experiencia en los Estados Unidos y Canadá sirvió, en gran medida y a partir del cambio de siglo, como base para corrientes de planificación más sostenibles e inclusivas. Ejemplos de ello son el llamado new urbanism o las prácticas de desarrollo compacto como el smart growth o los transit-oriented developments (TOD) (Downs, 2005; Hirt y Zahm, 2012).

Sin embargo, las teorías de Jacobs han tenido menos influencia en otras partes del mundo. Esto es especialmente claro en el contexto mediterráneo (Schubert, 2014), a pesar de que los atributos urbanos que la autora valoraba se corresponden, en gran parte, con los de las ciudades de estas latitudes, caracterizadas tradicionalmente por su carácter compacto, diverso y con un intenso uso del espacio público (Martín-Ramos, 2012; Morelli y Salvati, 2010; Pallares-Barbera et al., 2011; Salvati et al., 2013). No obstante, las ideas de Jacobs sí han tenido cierta influencia en este contexto en esferas más allá de la planificación urbana, especialmente en términos de movimientos sociales y trabajos teóricos críticos, ejemplificado en Barcelona por la organización Col-lectiu Punt 6 y su reivindicación de conceptos como el urbanismo feminista y los espacios de la vida cotidiana, acuñados bajo una clara influencia jacobsiana (Ciocoletto, 2014a; 2014b; Muxí Martínez et al., 2011).

A partir de la segunda mitad del siglo XX, las ciudades mediterráneas tradicionales, con su inherente vitalidad en el espacio público, se han ido transformando en formas urbanas desarrolladas bajo el paradigma de la modernidad, con menores densidades, mayores grados de fragmentación morfológica y social, y con un incremento del aislamiento de las funciones urbanas y de la dependencia de las infraestructuras viarias de transporte de alta capacidad (García-Palomares, 2010; Méndez, 2009; Salvati et al., 2015). Estas apuestas territoriales se traducen en distintos modelos de movilidad que, en la vida cotidiana, tienen un efecto directo en el modo en que la ciudadanía realiza sus desplazamientos diarios (Miralles-Guasch, 2002; Miralles-Guasch et al., 2018). Por un lado, mientras que los corrientes modernos del planeamiento urbano, a los que Jacobs se enfrentaba, primaban el vehículo privado, la velocidad y la lejanía, el discurso de la neoyorquina nos sitúa en la vida de barrio, 
el caminar cotidiano y la proximidad (Mateu et al., 2017; Miralles-Guasch y Marquet, 2013).

En este contexto, el objetivo de este estudio es evaluar la ciudad de Barcelona a través de las ideas de Jane Jacobs y relacionarlas con los distintos patrones de movilidad cotidiana de sus residentes. Para ello, en una primera parte se pretende valorar las condiciones que Jacobs consideraba necesarias para garantizar la vitalidad urbana en los distritos y barrios de Barcelona, de una forma cuantitativa y detallada. En segundo lugar, se analizan los principales patrones de movilidad cotidiana de las personas residentes en Barcelona en relación con los niveles de vitalidad de su barrio de residencia, con especial atención al caminar cotidiano. El artículo se estructura del siguiente modo: en el siguiente apartado se repasan las principales condiciones para la vitalidad según Jacobs y la relevancia de lo que ella dio a conocer como el «ballet de la acera»; a continuación, se presenta un apartado metodológico donde se describen las variables utilizadas y los cálculos realizados, y en el último apartado se muestran los resultados, que se discuten en una sección de conclusión.

\section{Condiciones para la vitalidad urbana y el «ballet de la acera»}

A través de su particular visión de la ciudad, Jane Jacobs se enfrentó a los patrones dominantes de planificación urbana de mitades del siglo XX, ejemplificados mediante figuras como la del ingeniero Robert Moses en Nueva York. En un momento en que la ortodoxia del planeamiento abordaba los desafíos urbanos exclusivamente desde los postulados modernos del urbanismo, basados en proyectos infraestructurales de gran magnitud y planes de segregación funcional (Corbusier, 1933; Hirt, 2016), Jacobs defendió la escala de la calle y del barrio y reubicó al individuo y la experiencia humana en el centro del debate. Así, Jacobs reivindicaba la vitalidad urbana como la característica básica para que las ciudades funcionaran, y para ello se propuso analizar las ciudades en la vida real, con el objetivo de entender qué principios podrían promover (o en su caso mantener) la vitalidad urbana o, por el contrario, qué prácticas la desincentivarían (Jacobs, 1961). Para ello, partió de cuatro condiciones complementadas con dos indicadores accesorios (Sung et al., 2015).

Según Jacobs, la primera condición para poder evaluar la vitalidad urbana es la diversidad, que en el ámbito académico se ha estudiado como la mezcla de los usos urbanos (Christian et al., 2011). Para Jacobs, la vitalidad es la suma de distintas diversidades, la de usos, propósitos y personas distintas en un espacio común y en diferentes momentos del día. En segundo lugar, Jacobs argumenta que la vitalidad requiere de una morfología urbana determinada, con manzanas más reducidas, que presente un número suficiente de intersecciones y esquinas que den lugar a una mayor oportunidad de contacto entre las personas. Esta morfología permite no solo una mayor diversidad de recorridos, y con ellos más interacción social, sino también un mayor 
dinamismo económico derivado de una mayor proximidad entre agentes (Knudsen et al., 2008). Paralelamente, Jacobs hace hincapié de forma recurrente en la necesidad de diseñar espacios urbanos a escala humana, en los que la persona se sienta el centro (Gehl, 2013; Whyte, 1980), en oposición a las morfologías diseñadas a vista de pájaro propias del movimiento moderno (Corbusier, 1933). Con el objetivo de garantizar que un barrio acoja distintos tipos de población y distintas rentas económicas, consideradas características básicas para la vitalidad urbana, Jacobs indica, en tercer lugar, la necesidad de promover un entorno construido que contenga cierta mezcla entre edificios de distintas características y edades. En este sentido, defiende que un barrio no solo necesita mixtura de tipos de edificios, sino también y específicamente habla de la necesidad conservar y preservar los inmuebles antiguos. Como cuarta condición, a lo largo de todo su relato Jacobs expone que la densidad es un elemento clave. En este sentido, considera que la concentración de edificios, de residencias y, fundamentalmente, de población es una condición elemental para la vida urbana. Con esta idea, Jacobs hace referencia no solo a la necesidad de que exista densidad residencial a modo de masa crítica, sino también al hecho de que la presencia de personas residentes es, a su vez, un elemento relevante para la atracción de actividad flotante.

A estas cuatro condiciones básicas de vitalidad urbana, se le pueden añadir dos elementos accesorios. En primer lugar, Jacobs hace referencia de forma transversal a la necesidad de garantizar la accesibilidad, es decir, que las personas residentes se puedan mover cómodamente por la ciudad sin depender del transporte privado. Esta fue una respuesta clara a la lógica predominante en la planificación del transporte del momento, que planteaba las infraestructuras para los automóviles como solución hegemónica (Foster, 1981). En segundo lugar, Jacobs dedica un capítulo entero a reflexionar sobre el efecto nocivo de lo que ella define como los «elementos de frontera». Este es el caso de las autopistas o los ferrocarriles en superficie, de los grandes edificios que no ofrecen una interacción con la calle por no tener puertas de entrada/salida suficientes, y de los grandes parques urbanos por generar efectos barrera en determinadas circunstancias. Para Jacobs, estos debían considerarse como elementos nocivos en la ciudad, al generar un efecto succionador de la vida en la calle dada su elevada impermeabilidad.

Desde el punto de vista de la movilidad cotidiana, la vitalidad urbana en la obra de Jacobs presenta una clara relación con el modo en que las personas utilizan la ciudad. Jacobs defendió la idea de que una intensa vida en la calle constituía el núcleo de lo que se entiende por urbanidad, lo que la autora definió de forma elegante como el «ballet de la acera» (Jacobs, 1961). Es por este motivo que los estudios que han tratado de llevar a la práctica las ideas teóricas de Jacobs han relacionado las condiciones de la vitalidad urbana con los patrones del caminar cotidiano a distintas escalas urbanas (Nadai et al., 2016; Delclòs-Alió et al., 2018; Sulis et al., 2018; Sung et al., 2013, 2015; Sung y Lee, 2015). Esto está estrechamente relacionado con la corriente académica 
que vincula el entorno construido y los desplazamientos a pie (ValenzuelaMontes y Talavera-García, 2015), tanto desde la óptica de un planeamiento urbano que garantice una mayor accesibilidad (Arranz-López et al., 2017; Southworth, 2005) así como las reflexiones proporcionadas por el urbanismo feminista, que ponen de claro relieve el acento en la vida cotidiana y en la dimensión del barrio.

Por último, las ideas de Jacobs nos remarcan, una y otra vez, la importancia del barrio como unidad de análisis. En este contexto, la vitalidad urbana no debería explicarse tan solo bajo el paradigma de la ciudad caminable (Forsyth, 2015; Marquet y Miralles-Guasch, 2015; Southworth, 2005), sino de una ciudad accesible en un radio de acción corto, como bien argumenta Jacobs. Este concepto es el que se ha definido como «proximidad», referido a aquellos desplazamientos cotidianos que se realizan a pie y en menos de diez minutos (Marquet y Miralles-Guasch, 2014).

\section{Metodología}

\subsection{Metodología para el análisis de la vitalidad urbana en Barcelona}

El análisis de la vitalidad urbana según Jane Jacobs se centra en este trabajo en Barcelona, una ciudad de 1.620.809 habitantes en una superficie de $101,35 \mathrm{~km}^{2}$ (Idescat, 2017). Es una de las urbes más densas de Europa (aprox. $16.000 \mathrm{hab} . / \mathrm{km}^{2}$ ), como consecuencia de un elevado nivel de urbanización (García, 2016), junto a unas tramas compactas ejemplificadas por el Eixample y también por los barrios anteriores a Cerdà. Administrativamente, la capital catalana se divide en 10 distritos y 73 barrios, utilizados en este trabajo como unidades territoriales de análisis (figura 1).

Con el objetivo de aplicar las ideas de Jane Jacobs en Barcelona, estas se han sintetizado en un total de seis condiciones básicas para la vitalidad urbana. De este modo, se analizarán los siguientes indicadores: Concentración, Diversidad, Oportunidad de contacto, Edad de los edificios, Accesibilidad y Elementos de frontera. Estas condiciones se han construido sobre la base de distintas combinaciones de un total de 11 variables, transformadas previamente a su correspondiente puntuación $\mathrm{Z}$ para ser comparables en términos de unidades (Frank et al., 2010). Las seis condiciones resultantes han sido ponderadas, entendiendo las cuatro primeras como las ideas básicas para la vitalidad urbana de Jacobs, mientras que las dos últimas son complementarias. Mediante esta metodología, se sintetizan estas seis ideas en el llamado índice Jane.

Los valores de las condiciones por sí mismas como de su síntesis se han calculado en una malla ortogonal constituida por celdas de $100 \times 100 \mathrm{~m}$, con el fin de homogeneizar la resolución espacial original de las distintas fuentes de datos. Esta metodología se encuentra descrita de forma sintética en la figura 2. Posteriormente y con el objetivo de llevar el análisis a las escalas territoriales propuestas por Jacobs, se ha resumido la información resultante mediante el cálculo de la media de cada indicador y del índice final tanto en el distrito como en el barrio. 
Figura 1. Mapa de distritos y barrios de Barcelona

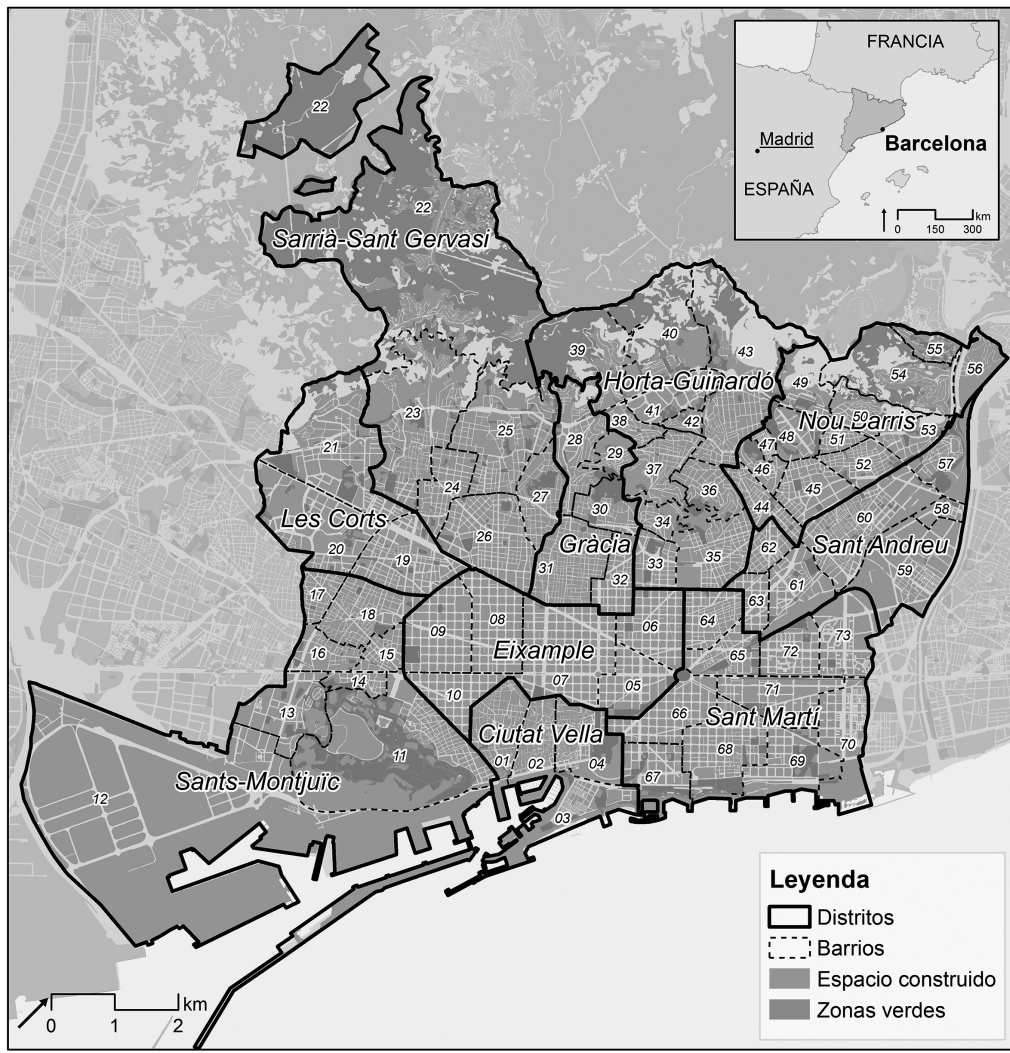

Distritos y barrios en Barcelona

\begin{tabular}{l}
\hline Ciutat Vella \\
\hline 01 el Raval \\
02 \\
03 el Bari Gótic \\
03 larceloneta
\end{tabular}

04 Sant Pere, Santa Caterina i la Ribera

\section{5 el Fort Pienc}

66 la Sagrada Familia

07 la Dreta de l'Eixamole

08 l'Antiga Esquerra de l'Eixample

Sa Nova Esquerra de lEixample

10 Sant Antoni

\begin{tabular}{l} 
Sants-Montiü̈c \\
\hline 11 el Poble Sec - Parc Montiuíc \\
17 Sants - Badal
\end{tabular}

12 la Marina del Prat Vermell - Zona F. 18 Sants

13 la Marina de Port

14 la Font de la Guatl

15 Hostafrancs

16 la Bondeta

19 les Corts

la Matemitat i Sant Ram

21 Pedralbes

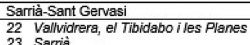

23 Saria

24 les Tres Torres

25 Sant Gervasi - la Bonanova

26 Sant Gervasi - Galvany

Gràci

28 Vallcarca $j$ els Penitents

29 el Coll

30 la Salut

32 el Camp d'en Grassot $i$ Gràcia Nova

Fuente: elaboración propia.

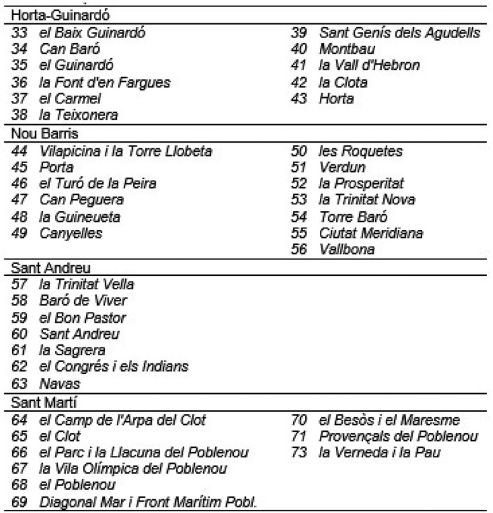

Documents d'Anàlisi Geogràfica 2021, vol. 67/1 
Figura 2. Esquema metodológico en la construcción de las condiciones para la vitalidad urbana

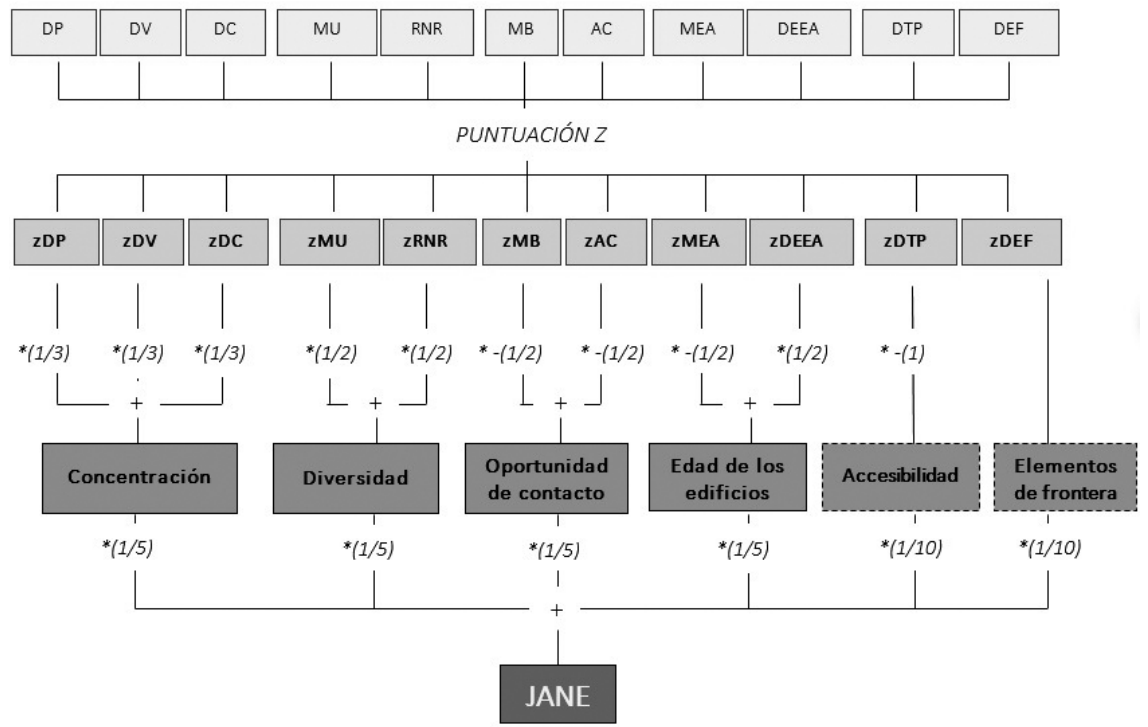

DP = Densidad de población (habitantes $/ \mathrm{km} 2$ ); DV = Densidad de viviendas (viviendas $/ \mathrm{km} 2$ ); DC = Densidad construida (área construida/área catastral); $M U$ = Mezcla de usos (, en la que pi se refiere a la proporción del uso i en cada edificio, considerando todos los usos, y en la que n es el número total de usos; RNR = Índice residencial-no-residencial (donde Resi se refiere al uso residencial, y Non-Resi se refiere a los usos no residenciales en cada edificio); $M B=$ Dimensión del bloque (m2); $A C=$ Ancho de calle (m); MEA = Media de las edades de los edificios; DEEA = Desviación estándar de las edades de los edificios; DTP = Distancia a la parada de transporte público más cercana $(\mathrm{m})$; DEF = Distancia al elemento de frontera más cercano $(\mathrm{m}) ; \mathrm{z}=$ Puntuación $\mathrm{Z}$.

Fuente: elaboración propia.

\subsection{Indicadores de movilidad cotidiana en Barcelona}

Con el objetivo de relacionar la vitalidad urbana en el barrio con los principales patrones de movilidad cotidiana de las personas residentes en Barcelona, la segunda parte del análisis de este trabajo se sirve de datos extraídos de la Encuesta de Movilidad en Días Laborables de 2015 (EMEF'15). Esta es una encuesta representativa, realizada de forma anual, para toda la Región Metropolitana de Barcelona, con una muestra de 2.816 individuos para el municipio de Barcelona (el perfil de la muestra se presenta en la tabla 1). En Barcelona, en días laborables, alrededor de un $92 \%$ de la población se desplaza por algún motivo, con aproximadamente 5,4 millones de viajes cotidianos, lo que representa una media de 4,28 desplazamientos por persona y día.

Entre la distinta información disponible en la encuesta, la vitalidad urbana en el barrio se ha relacionado con los siguientes indicadores de movilidad cotidiana. En primer lugar, se analiza la proporción de inmovilidad, es decir, el porcentaje de población que el día de la encuesta no salió de casa, el número 
Tabla 1. Datos básicos de la muestra de EMEF'15 para el municipio de Barcelona

\begin{tabular}{lrr}
\hline & $n$ & $\%$ \\
\hline Total & 2.816 & 100,0 \\
\hline Tipo de población & 2.570 & \\
$\quad$ Móvil no profesional & 32 & 1,3 \\
$\quad$ Móvil profesional & 214 & 7,6 \\
Inmóvil & & \\
\hline Género & 1.310 & 46,5 \\
$\quad$ Hombre & 1.506 & 53,5 \\
Mujer & & \\
\hline Edad & 474 & 16,8 \\
De 16 a 30 & 1.003 & 35,6 \\
De 31 a 50 & 677 & 24,0 \\
De 51 a 65 & 662 & 23,5 \\
Más de 65 & & \\
Situación profesional & 1.477 & 52,4 \\
Activo ocupado & 223 & 7,9 \\
Activo en paro & 209 & 7,4 \\
Estudiante & 863 & 30,6 \\
Jubilado o prejubilado & 45 & 1,6 \\
Otros & & \\
\hline
\end{tabular}

Fuente: elaboración propia.

de desplazamientos por persona y día y el tiempo diario de desplazamiento en minutos, y se suma el conjunto de desplazamientos de la persona. En segundo lugar, se presenta la distribución modal media de cada individuo, es decir, el porcentaje de uso habitual de cada medio de transporte, considerando el ir a pie, el transporte público (incluyendo todas las opciones en la ciudad) y el transporte privado (con todo tipo de vehículos de propiedad individual). En tercer lugar y considerando que el principal interés del análisis es con relación a la movilidad a pie, se presentan también variables específicas para este tipo de desplazamiento: frecuencia con la que las personas entrevistadas dicen caminar de forma cotidiana y dos indicadores más, uso de los desplazamientos de proximidad y relación con la actividad fisica recomendada al día. Por desplazamientos de proximidad, se entienden aquellos realizados a pie y con duraciones inferiores a los diez minutos, según estudios previos (Marquet y Miralles-Guasch, 2015; Miralles-Guasch y Marquet, 2013). Por otro lado y considerando que la Organización Mundial de la Salud recomienda 30 minutos de actividad física moderada al día (OMS, 2010), se propone usar el umbral de los 30 minutos a pie al día como referencia para explorar si existen diferencias en el tiempo caminado diario en términos de vitalidad urbana del barrio de residencia.

Esta parte del análisis se ha basado en tablas de contingencia, usando el estadístico de Chi-cuadrado para identificar relaciones estadísticamente relevantes y valores específicos significativamente superiores o inferiores a lo esperado. 


\section{Resultados}

El análisis de la vitalidad urbana en Barcelona se estructura en dos partes. En primer lugar, se presentan por distritos los resultados obtenidos en cada una de las condiciones básicas definidas por Jacobs, así como su puntuación final en el índice Jane también por barrios. En segundo lugar, se relacionan dichos valores de los barrios con los que se consideran los principales indicadores de la movilidad cotidiana de sus residentes, con una especial atención a los desplazamientos a pie.

\subsection{Vitalidad urbana en distritos y barrios de Barcelona}

Dadas sus características urbanas, Barcelona es en general una ciudad vital de acuerdo con las lógicas de Jane Jacobs. No obstante, la vitalidad urbana no es homogénea, tal y como muestra la tabla 2. Mientras que los distritos centrales de la ciudad (Ciutat Vella, Eixample y Gràcia) tienen mayor vitalidad (con indicadores Jane alrededor de entre 0,59 y 0,67 ), los distritos de su alrededor presentan valores menores, donde Les Corts se presenta como el espacio de menor vitalidad de Barcelona, con tan solo un 0,08 en la escala de Jane. Respecto a distritos, se puede hablar de una lógica territorial de la vitalidad, de centro-periferia.

Estas diferencias se explican por distintas combinaciones de las seis condiciones definidas por Jane Jacobs. Barcelona es una ciudad densa en su conjunto, pero, a pesar de ello, los distritos centrales, y especialmente el ensanche de Cerdà, destacan por encima del resto en la condición referida a la Concentración. Los barrios más alejados del centro y con una mayor proporción de morfología dispersa o de baja densidad (Horta-Guinardó, Les Corts y Sarrià-Sant Gervasi) son los que, consecuentemente, presentan menores valores en este campo. Del mismo modo que en el caso anterior, existen diferencias en cuanto

Tabla 2. Condiciones para la vitalidad urbana en los distritos de Barcelona (valores medios)

\begin{tabular}{|c|c|c|c|c|c|c|c|}
\hline & Concentración & Diversidad & $\begin{array}{l}\text { Oportunidad } \\
\text { de contacto }\end{array}$ & $\begin{array}{c}\text { Edad de } \\
\text { Ios edificios }\end{array}$ & Accesibilidad & $\begin{array}{l}\text { Elementos } \\
\text { de frontera }\end{array}$ & Jane \\
\hline Ciutat Vella & 0,85 & 0,07 & 0,13 & 1,82 & 0,36 & 0,25 & 0,64 \\
\hline Eixample & 1,77 & 0,27 & $-0,87$ & 1,25 & 0,39 & 1,46 & 0,67 \\
\hline Gràcia & 1,18 & 0,32 & 0,23 & 0,75 & 0,40 & 0,54 & 0,59 \\
\hline Horta-Guinardó & 0,66 & 0,24 & $-0,07$ & 0,45 & 0,38 & $-0,08$ & 0,29 \\
\hline Les Corts & 0,75 & 0,04 & $-0,59$ & 0,12 & 0,37 & $-0,20$ & 0,08 \\
\hline Nou Barris & 0,98 & 0,15 & 0,07 & 0,07 & 0,40 & $-0,24$ & 0,27 \\
\hline Sant Andreu & 0,90 & 0,03 & $-0,40$ & 0,27 & 0,36 & 0,12 & 0,21 \\
\hline Sant Martí & 0,91 & 0,01 & $-0,58$ & 0,27 & 0,36 & 0,44 & 0,20 \\
\hline Sants-Montjuïc & 1,04 & 0,13 & $-0,27$ & 0,57 & 0,35 & $-0,02$ & 0,33 \\
\hline Sarrià-Sant Gervasi & 0,59 & 0,48 & 0,03 & 0,52 & 0,35 & $-0,03$ & 0,35 \\
\hline Barcelona & 0,95 & 0,17 & $-0,22$ & 0,51 & 0,37 & 0,18 & 0,34 \\
\hline
\end{tabular}

Fuente: elaboración propia. 
a la Diversidad, referida a los usos urbanos. En este caso, se pueden dibujar a grandes rasgos dos grandes bloques: un primer bloque de elevada diversidad configurado por los distritos del Eixample, Gràcia, Horta-Guinardó y SarriàSant Gervasi, y otro con el resto de los distritos, con valores muy inferiores de mixtura de usos.

La lógica de las dos siguientes condiciones, Oportunidad de contacto y Edad de los edificios, habla de la historia de la ciudad de Barcelona. Aquellos distritos que contienen las zonas más antiguas de la ciudad son, a la vez, los que disponen de una trama urbana que permite un mayor contacto, fundamentalmente debido a su malla irregular, compacta y de bloques pequeños, y, por otro lado, las que presentan mayores valores en cuanto a la diversidad de edades de la edificación. El caso del ensanche es quizás particular, debido a que, a pesar de ser en su parte central una de las zonas más antiguas fuera de los cascos, su morfología basada en calles anchas y bloques de aproximadamente $120 \mathrm{~m}$ lo puntúa bajo en este indicador.

Por lo que se refiere a las dos condiciones complementarias, la amplia cobertura de transporte público de esta gran ciudad tiene como consecuencia que el indicador de Accesibilidad sea prácticamente igual en todos los distritos. Por otro lado, y por último, el rol de los Elementos de frontera es particularmente importante en aquellas zonas en contacto con grandes infraestructuras de comunicación en superficie, como pueden ser Les Corts o Nou Barris, mientras que los tres distritos centrales y el distrito de Sant Martí quedan más protegidos del efecto nocivo de dichos elementos.

No obstante, es en el contexto del barrio donde Jacobs recogía sus impresiones y, en consecuencia, donde la vitalidad urbana debe analizarse con mayor detalle. En este sentido, la figura 3 muestra el detalle por barrios, unidades administrativas menores que los distritos, y aquí se rompen algunos patrones observados anteriormente en las unidades mayores, los distritos. A primera vista, se dibuja una vez más la lógica de centro y periferia, pero con matices interesantes a destacar. Por ejemplo, en distritos como Sants-Montjuïc o Sant Andreu, el mapa refleja la diferencia entre los cascos de estas antiguas localidades respecto a sus desarrollos más recientes o aquellas partes cercanas a infraestructuras de comunicación, o en el primer caso, la zona industrial alrededor de la colina de Montjuïc. Por otro lado, en los barrios al noreste del municipio (distritos de Horta-Guinardó y Nou Barris), se distinguen a su vez las partes más urbanas, densas y diversas, cercanas al centro, de las zonas en contacto con Collserola, con un mayor peso del tejido disperso o de baja densidad.

A modo de ejemplo de los contrastes entre barrios, es de especial interés destacar los patrones que dibujan los barrios de Sant Martí. Alrededor de los barrios de vitalidad media e incluso alta correspondientes a la parte antigua del Poblenou, en el centro, y los barrios del Camp de l'Arpa y del Clot, emergen una serie de barrios de vitalidad baja. Por un lado, en el contacto con el ensanche, el barrio del Parc i la Llacuna del Poblenou se corresponde con antiguas zonas industriales con menor densidad residencial, cuyo paisaje morfológico y social ha sufrido importantes cambios debido a los nuevos desarrollos rela- 
Figura 3. Mapa de la vitalidad urbana en los barrios de Barcelona

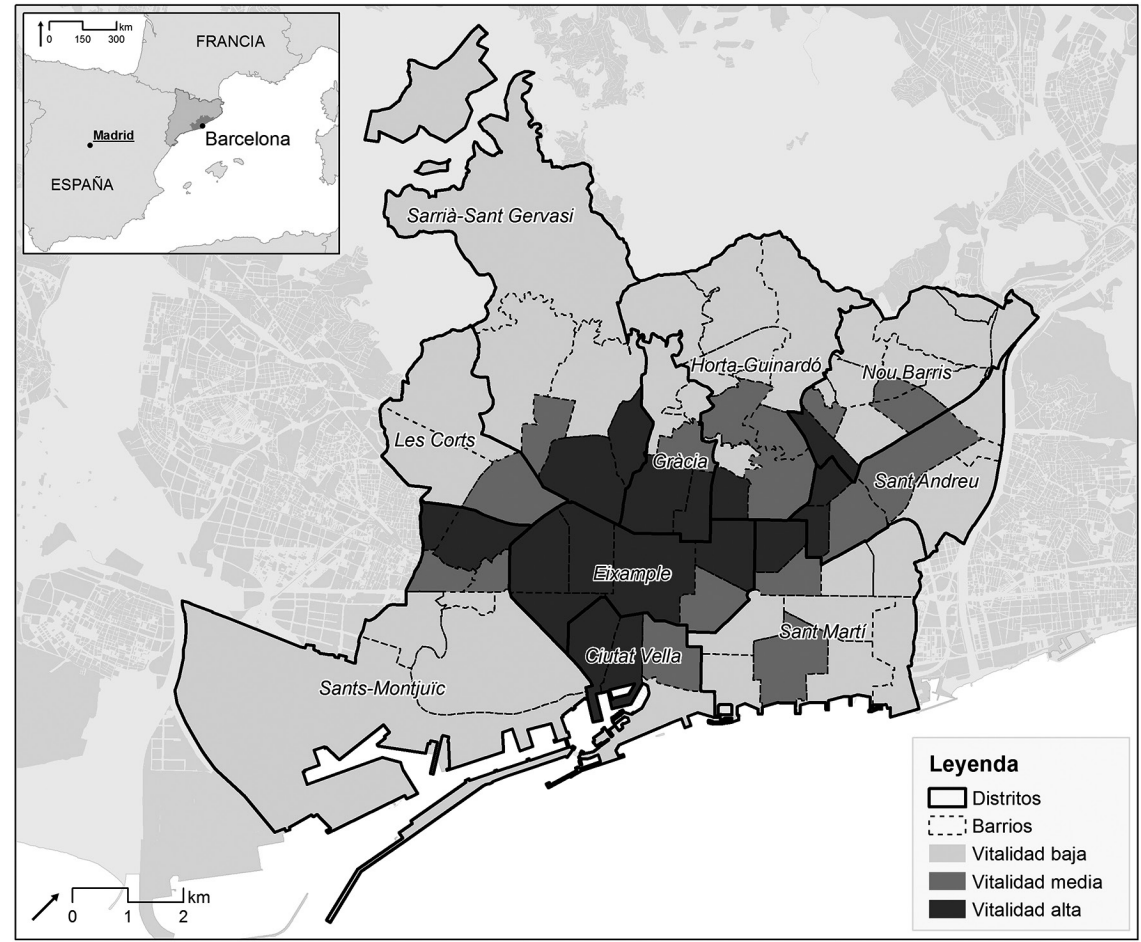

Fuente: elaboración propia.

cionados con las empresas tecnológicas y el proyecto del 22@ (Casellas, 2007; Paül i Agustí, 2017). Por otro lado, destacan también los casos de la Vila Olímpica y el Fòrum, a ambos lados del centro del Poblenou, también fruto de procesos de renovación urbana derivados de los Juegos Olímpicos de 1992 y del Fórum de las Culturas de 2004, respectivamente.

\subsection{Vitalidad urbana en los barrios y patrones de movilidad cotidiana en Barcelona}

Si el barrio fue la escala del análisis para Jane Jacobs, el modo en que sus residentes se desplazan puede ser considerado el reflejo de la vitalidad urbana. A continuación, se detalla la relación entre la vitalidad del barrio de residencia de las y los barceloneses con sus principales patrones de movilidad cotidiana.

En la tabla 3, se observa que dos de los tres indicadores básicos de movilidad cotidiana son similares entre las personas residentes en los tres tipos de barrio, con alrededor de 4,2-4,3 desplazamientos per cápita al día y alrededor 91-93 minutos dedicados cada día a moverse. No obstante, en el primer indi- 
Tabla 3. Vitalidad urbana del barrio de residencia e indicadores generales de movilidad cotidiana

\begin{tabular}{lccc}
\hline Vitalidad del barrio & $\begin{array}{c}\text { Inmovilidad } \\
\text { (\% de la categoría) }^{\text {a }}\end{array}$ & $\begin{array}{c}\text { Desplazamientos } \\
\text { por persona al día }\end{array}$ & $\begin{array}{c}\text { Tiempo de viaje diario } \\
\text { (minutos) }\end{array}$ \\
\hline Baja $(<0,38)$ & $8,9^{* \star}$ & 4,20 & 91,83 \\
Media $(0,38-0,58)$ & 8,1 & 4,28 & 93,22 \\
Alta $(>0,58)$ & $5,8^{\star}$ & 4,37 & 92,90 \\
Barcelona & 7,6 & 4,28 & 92,62 \\
\hline
\end{tabular}

a. Test: $\mathrm{Chi}^{2} \mathrm{sig}<0.005$. Test de residuos corregidos

*Valores significativamente inferiores

**Valores significativamente superiores

Fuente: elaboración propia a partir de EMEF'15.

Tabla 4. Vitalidad urbana del barrio de residencia y distribución modal cotidiana

\begin{tabular}{lcccc}
\hline & \multicolumn{4}{c}{ Distribución modal } \\
\cline { 2 - 4 } Vitalidad del barrio & A pie (\%) & Transp. público (\%) & Transp. privado (\%) & Total (\%) \\
\hline Baja $(<0,38)$ & $45,7^{*}$ & 30,5 & $23,7^{\star *}$ & 100 \\
Media $(0,38-0,58)$ & 49,8 & 30,4 & $19,9^{\star}$ & 100 \\
Alta $(>0,58)$ & $51,8^{\star *}$ & $28,4^{*}$ & $19,8^{\star}$ & 100 \\
\hline Barcelona & 49,1 & 29,7 & 21,2 & 100 \\
\hline
\end{tabular}

a. Test: $\mathrm{Chi}^{2} \mathrm{sig}<0.005$. Test de residuos corregidos

*Valores significativamente inferiores

**Valores significativamente superiores

Fuente: elaboración propia a partir de EMEF'15.

cador sí existe una diferencia significativa: en conjunto, las personas residentes en barrios muy vitales salen más de casa que las que residen en barrios de media o baja vitalidad (el 5,8\% de inmovilidad y alrededor del $9 \%$ de inmovilidad, respectivamente).

En segundo lugar, cabe destacar las diferencias entre tipos de barrio por lo que se refiere a la distribución modal de sus residentes (tabla 4), es decir, el grado en el que se usa de forma cotidiana cada modo de transporte. A pesar de que Barcelona sea un municipio en el que ir a pie es el principal modo de transporte, aquellas personas que residen en barrios de elevada vitalidad presentan un porcentaje significativamente superior de sus desplazamientos realizados caminando $(51,8 \%)$, en detrimento del uso del transporte público y del privado, en los que se observan resultados inferiores a la media. De forma relacionada pero contrapuesta, entre aquellos que residen en entornos de baja vitalidad, el uso del transporte privado es significativamente mayor $(23,7 \%)$.

Estas pautas se confirman con los resultados referidos al modo en que las personas encuestadas reportan la frecuencia con la que caminan de forma cotidiana (tabla 5). Una vez más, mientras que en general la mayor parte de la población de Barcelona camina de forma cotidiana, en las respuestas En ocasiones (33,89\%), A menudo (36,43\%) y Siempre (12,13\%), existen también 
Tabla 5. Vitalidad urbana del barrio de residencia y predisposición para desplazarse a pie

\begin{tabular}{llccccc}
\hline & \multicolumn{5}{c}{ Frecuencia con la que se camina de forma cotidiana $^{\text {a }}$} \\
\cline { 2 - 7 } Vitalidad del barrio & $\begin{array}{c}\text { Nunca } \\
(\%)\end{array}$ & $\begin{array}{c}\text { Raramente } \\
(\%)\end{array}$ & $\begin{array}{c}\text { En ocasiones } \\
(\%)\end{array}$ & $\begin{array}{c}\text { A menudo } \\
(\%)\end{array}$ & $\begin{array}{c}\text { Siempre } \\
(\%)\end{array}$ & $\begin{array}{c}\text { Total } \\
(\%)\end{array}$ \\
\hline Baja $(<0,38)$ & $4,71^{\star *}$ & $16,07^{* *}$ & 31,86 & $33,52^{*}$ & 13,85 & 100 \\
Media $(0,38-0,58)$ & 3,80 & 13,95 & 35,50 & 34,71 & 12,04 & 100 \\
Alta $(>0,58)$ & $2,17^{\star}$ & $11,85^{*}$ & 34,54 & $41,04^{\star *}$ & 10,40 & 100 \\
\hline Barcelona & 3,57 & 13,99 & 33,89 & 36,43 & 12,13 & 100 \\
\hline
\end{tabular}

a. Test: $\mathrm{Chi}^{2} \mathrm{sig}<0.005$. Test de residuos corregidos

*Valores significativamente inferiores

${ }^{*}$ Valores significativamente superiores

Fuente: elaboración propia a partir de EMEF'15.

diferencias significativas entre residentes de distintos barrios en función de su vitalidad. Entre aquellos que residen en un barrio de elevada vitalidad, el porcentaje de gente que anda $A$ menudo es significativamente superior que en los otros barrios. De forma contrapuesta, entre aquellos que residen en barrios de baja vitalidad, la proporción que camina Nunca $(4,71 \%)$ y Raramente $(16,07 \%)$ es significativamente superior que en los barrios de media y alta vitalidad urbana.

Por último, en la tabla 6 se presentan los resultados de dos indicadores específicos del ir a pie cotidiano: el uso de la proximidad (desplazamientos a pie de menos de diez minutos) y la parte de la población que consigue superar los 30 minutos a pie recomendados por la Organización Mundial de la Salud. En primer lugar, de los 4,28 desplazamientos que cada persona residente en la ciudad realiza de media al día, una parte considerable de estos corresponden a desplazamientos de proximidad (1,25 desplazamientos, $28,2 \%$ del total). No obstante, estos indicadores difieren una vez más en función de la vitalidad del barrio de residencia: aquellas personas que viven en entornos muy vitales

Tabla 6. Vitalidad urbana del barrio de residencia, uso de la proximidad y relación con el caminar recomendado al día (30 minutos)

\begin{tabular}{|c|c|c|c|}
\hline \multirow[b]{2}{*}{ Vitalidad del barrio } & \multicolumn{2}{|c|}{ Desplazamientos de proximidad cotidianos } & \multirow[b]{2}{*}{$\begin{array}{l}\text { Residentes que superan } \\
\text { los } 30 \text { min a pie al día }(\%)^{b}\end{array}$} \\
\hline & Despl. por persona & $\begin{array}{l}\% \text { respecto a todos } \\
\text { los desplazamientos }\end{array}$ & \\
\hline Baja $(<0,38)$ & 1,15 & $26,3^{*}$ & $36,3^{*}$ \\
\hline Media $(0,38-0,58)$ & 1,25 & 28,2 & 42,3 \\
\hline Alta $(>0,58)$ & 1,36 & $30,1^{\star *}$ & 40,9 \\
\hline Barcelona & 1,25 & 28,2 & 39,7 \\
\hline
\end{tabular}

a, b. Test: $\mathrm{Chi}^{2} \mathrm{sig}<0.005$. Test de residuos corregidos

*Valores significativamente inferiores

**Valores significativamente superiores

Fuente: elaboración propia a partir de EMEF'15. 
pueden realizar una parte significativamente mayor de su movilidad cotidiana en desplazamientos de proximidad $(30,1 \%)$. Por otro lado, mientras que de media alrededor del $40 \%$ de la población de Barcelona consigue superar los 30 minutos a pie recomendados por la OMS, entre las personas residentes en barrios de baja vitalidad esta proporción es ligeramente inferior (36,3\%).

\section{Conclusión}

Este estudio tiene como objetivo la aplicación y la evaluación de las ideas de Jane Jacobs en la ciudad de Barcelona. Para ello, en una primera parte se han analizado, por distritos y barrios, las condiciones que Jacobs consideraba necesarias para garantizar la vitalidad urbana. En segundo lugar, se han relacionado dichos indicadores de vitalidad por barrios con los principales patrones de movilidad cotidiana de sus residentes, con una especial atención al caminar cotidiano y las dinámicas de proximidad.

Barcelona, como ejemplo de ciudad mediterránea, puede considerarse, a grandes rasgos, una urbe de una elevada vitalidad, aunque con diferencias internas. Esto es de especial interés teniendo en cuenta que, como otras muchas ciudades mediterráneas, Barcelona combina su tradicional naturaleza vital con formas urbanas que solo se entienden bajo el paradigma de la modernidad, con menores densidades, menor diversidad y mayor peso de las infraestructuras de transporte de alta capacidad (Morelli y Salvati, 2010). Entre las distintas variables de Jane Jacobs, las condiciones en las que se han detectado más desigualdades son las referidas a la Concentración, la Oportunidad de contacto, la Edad de los edificios y la complementaria referida a los Elementos de frontera. Por otro lado, y a pesar de presentar algunas diferencias, la Diversidad y especialmente la Accesibilidad son considerablemente homogéneas en esta ciudad. La distinta combinación de estas variables tiene como consecuencia que los barrios con las características más vitales no solo se ubiquen en el centro del municipio, sino también en zonas más periféricas que no coinciden con las de los barrios históricos. Esto se relaciona principalmente con los esfuerzos realizados durante la década de 1980, en los años previos a los Juegos Olímpicos de 1992, para mejorar la calidad del entorno construido en toda la ciudad, a través de proyectos específicos de renovación urbana que, con el tiempo, han demostrado ser exitosos (Degen y García, 2012; Montoya, 2014). Ello implica que no solo los cascos antiguos y los centros urbanos pueden albergar vitalidad urbana, sino que desarrollos nuevos también pueden conseguirlo, en función de los parámetros considerados en su diseño (Cho y Kim, 2017).

Además, resulta de especial interés identificar las razones de la baja vitalidad de ciertos barrios, que obedecen a tres factores distintos. En primer lugar, son las zonas limítrofes de la ciudad, donde se ubican las áreas menos urbanizadas, como los parques de Collserola y Montjuïc, y las infraestructuras de transporte de gran capacidad. En segundo lugar, se trata de las morfologías constructivas menos vitales, como pueden ser los barrios de menor densidad del distrito de Les Corts o aquellos con una mayor proporción de polígonos de vivienda en 
altura, desarrollados entre los años sesenta y setenta del siglo pasado, y ubicados principalmente a lo largo del eje Besós. En tercer lugar y con un especial interés, son barrios que han experimentado proyectos de transformación urbana reciente y a gran escala, todos ubicados en el distrito de Sant Martí. Es el caso, por ejemplo, del barrio de la Vila Olímpica, que, a diferencia de los anteriormente mencionados proyectos preolímpicos, no parece recoger las condiciones de Jacobs para la vitalidad urbana. Es también el caso de grandes proyectos como Diagonal Mar y el espacio relacionado a la celebración del Fórum de las Culturas en 2004. Aquí, estas operaciones han sido fuertemente criticadas, tanto por los vecinos como por expertos, dada la ruptura que supone su morfología con la de los barrios preexistentes. Del mismo modo, las condiciones para la vitalidad urbana no parecen estar presentes en los principales barrios en los que, hasta la fecha, se ha desarrollado el proyecto del 22@, diseñado para constituir un distrito de innovación, con un importante proceso de atracción de empresas tecnológicas (Paül i Agustí, 2017).

Si bien los barrios de Barcelona presentan considerables diferencias por lo que se refiere a su potencial capacidad para ser entornos vitales, se ha detectado a su vez una clara relación con las pautas de movilidad cotidiana de sus residentes. Entre los indicadores globales de movilidad cotidiana, debe destacarse que en aquellos barrios donde se cumplen en mayor medida las condiciones defendidas por Jacobs, la población residente presenta una tasa de inmovilidad significativamente menor. En primer lugar, las características del entorno urbano están relacionadas con la población que en su día a día sale de casa, lo que refuerza la idea de que la forma urbana puede incidir en el riesgo de aislamiento social, en este caso evitarlo (Kent y Thompson, 2014).

En segundo lugar, se ha identificado una estrecha relación entre la vitalidad en el barrio y la relevancia del caminar cotidiano de sus residentes, tanto en su elección modal y como en su frecuencia. En estudios anteriores en los que se relaciona el entorno construido con el caminar cotidiano, la elección de la densidad urbana como única variable de análisis ha demostrado un efecto reducido en ciudades como Barcelona, donde esta característica urbana es bastante homogénea en casi toda la ciudad (Marquet y Miralles-Guasch, 2015). En cambio, un enfoque multidimensional como el que propuso Jacobs, reunido en el concepto de vitalidad urbana, sí parece tener una relación con la movilidad a pie. Una relación que también se manifiesta en sentido inverso, pues en aquellos barrios menos vitales y más alejados del modelo mediterráneo de ciudad, la dependencia del transporte motorizado, y especialmente del transporte privado, es superior.

En la misma línea, no solo son más importantes los desplazamientos a pie para las personas que viven en entornos vitales, sino que también existe una mayor relevancia de las dinámicas de proximidad. Aun teniendo en cuenta que la proximidad es relevante en toda Barcelona (Marquet y MirallesGuasch, 2014), dado su carácter compacto (Busquets, 2005) y policéntrico (Cerda Troncoso y Marmolejo Duarte, 2010), la utilización del índice de vitalidad propuesto por Jacobs muestra la relevancia de la escala del barrio 
para identificar esos desplazamientos cortos. En este sentido, los barrios vitales no solo muestran beneficios respecto a accesibilidad, con lo que significa para la inclusión social, sino que también son relevantes para la salud individual. Se demuestra una vez más, en la línea de otros trabajos recientes (Marquet et al., 2017), que los barrios caminables permiten estilos de vida más saludables, ejemplificados en este estudio por la proporción de población que consigue superar los 30 minutos recomendados de caminar cotidiano.

Este trabajo representa una interpretación del trabajo teórico de Jacobs en relación con su concepto de vitalidad urbana, a pesar de considerar la existencia de muchas otras que podrían y deberían realizarse. Entre ellas, en primer lugar, cabría destacar la necesidad de reinterpretar a Jane Jacobs en el contexto actual, con la inclusión de nuevas variables en el análisis, como los aspectos socioeconómicos o fenómenos con un elevado impacto urbano derivados precisamente de la calidad de vida en los barrios; como la turistificación de la ciudad, los procesos de gentrificación y su relación con la movilidad cotidiana (Blanco et al., 2014), así como las desigualdades sociales presentes a día de hoy en las ciudades. Esto es especialmente ejemplificado en el distrito de Ciutat Vella, uno de los entornos de mayor vitalidad potencial pero a la vez uno de los principales escenarios de problemáticas, como la substitución de la población como resultado indirecto de la mayor presión sobre la vivienda derivada del turismo de masas, así como el drama contemporáneo de los desahucios, según se ha evidenciado en estudios recientes (Gutiérrez y Domènech, 2018).

En segundo lugar, a pesar de que el escenario del análisis de Jacobs fuese el barrio en el que la población vive, es necesario analizar las características del espacio construido en el que tienen lugar los recorridos a pie cotidianos más allá del entorno residencial (Kwan, 2018). En tercer lugar y como principal limitación del estudio, este se ha focalizado en la población residente sin prestar atención a diferencias en las características personales que seguro podrían matizar los resultados aquí presentados. En este sentido, sería necesaria una segunda lectura e interpretación de los datos en términos de género, ya que esta variable es clave en el análisis tanto de la experiencia del espacio cotidiano (Muxí Martínez et al., 2011) y su necesaria lectura desde el punto de vista de la planificación urbana (Ortiz Escalante y Gutiérrez Valdivia, 2015) como también y especialmente en términos de los patrones de movilidad cotidiana (Maciejewska et al., 2020; Miralles-Guasch, 1998; Miralles-Guasch et al., 2015).

Para concluir, este trabajo muestra de qué modo la aplicación de las ideas de Jacobs a un caso concreto como el de Barcelona, permite analizar los efectos que generan distintas configuraciones urbanas. Por un lado, aquellas propias de la lógica moderna de planificación y, por otro, aquellas que defienden un uso efectivo del espacio público, ejemplificado, en este estudio, por la movilidad cotidiana a pie. En las últimas páginas de su libro de 1961, Jacobs hace hincapié en la necesidad de «diagnosticar qué condiciones generadoras de diversidad faltan ahí [en un determinado proyecto o barrio], si hay una carencia de usos primarios combinados, si las manzanas son demasiado grandes, si no 
hay suficiente mezcla de edad y tipo de los edificios, o si la concentración de población es insuficiente. Después, cualquiera de estas condiciones que se eche en falta ha de introducirse lo mejor posible, gradual y oportunamente» (Jacobs, 1961: 434). En este sentido, identificar en una ciudad concreta y con el detalle necesario aquellas áreas que son o no son vitales puede considerarse un instrumento básico para los distintos agentes urbanos implicados en el proceso de planificación. Pues no solo se necesitan políticas públicas dirigidas a la habitabilidad de la ciudad, sino también a promover la vitalidad de sus barrios.

\section{Referencias bibliográficas}

Arranz-López, A.; Soria-Lara, J. A.; López-Escolano, C. y Pueyo Campos, Á. (2017). «Retail Mobility Environments: A methodological framework for integrating retail activity and non-motorised accessibility in Zaragoza, Spain». Journal of Transport Geography, 58, 92-103. $<$ https://doi.org/10.1016/j.jtrangeo.2016.11.010>

BlanCO, J.; BosOER, L. y APAOlAZA, R. (2014). «Gentrificación, movilidad y transporte: aproximaciones conceptuales y ejes de indagación». Revista de Geografía Norte Grande, 58, 41-53.

BusqueTs, J. (2005). Barcelona: The Urban Evolution of a Compact City. Rovereto: Nicolodi.

CASELLAS, A. (2007). «Gobernabilidad, participación ciudadana y crecimiento económico: adaptaciones locales a estrategias globales». Scripta Nova. Revista Electronica de Geografiía y Ciencias Sociales, XI (243).

Cerda Troncoso, J. y Marmolejo Duarte, C. (2010). «De la accesibilidad a la funcionalidad del territorio: Una nueva dimensión para entender la estructura urbano-residencial de las áreas metropolitanas de Santiago (Chile) y Barcelona (España)». Revista de Geografía Norte Grande, 27 (46), 5-27. $<$ https://doi.org/10.4067/S0718-34022010000200001>

Cho, S. E. y KIM, S. (2017). «Measuring urban diversity of Songjiang New Town: A re-configuration of a Chinese suburb». Habitat International, 66, 32-41. <https://doi.org/10.1016/j.habitatint.2017.05.009>

Christian, H. E.; Bull, F. C.; Middleton, N. J.; Knuiman, M. W.; Divitini, M. L.; HoOper, P.; Amarasinghe, Anura K. y Giles-Corti, B. (2011). «How important is the land use mix measure in understanding walking behaviour? Results from the RESIDE study». The International Journal of Behavioral Nutrition and Physical Activity, 8, 55. $<$ https://doi.org/10.1186/1479-5868-8-55>

Ciocoletto, A. (2014a). Espais per a la vida quotidiana: Auditoria de Qualitat Urbana amb Perspectiva de Gènere. Col-lectiu Punt 6.

- (2014b). «Urbanismo para la vida cotidiana: herramientas de análisis y evaluación urbana a escala de barrio desde la perspectiva de género». TDX (Tesis Doctorals en Xarxa), 1-256. Recuperado de <http://hdl.handle.net/10803/275979>.

Corbusier, L. (1933). The Athens Charter. Nueva York: Grossman Publishers, 1973.

DEGEN, M. y GARCíA, M. (2012). «The Transformation of the "Barcelona Model": An Analysis of Culture, Urban Regeneration and Governance». International Journal of Urban and Regional Research, 36 (5), 1.022-1.038. <https://doi.org/10.1111/j.1468-2427.2012.01152.x> 
Delclòs-Alió, X.; Gutiérrez, A. y Miralles-Guasch, C. (2018). «The urban vitality conditions of Jane Jacobs in Barcelona: Residential and smartphone-based tracking measurements of the built environment in a Mediterranean metropolis». Cities, In Press. <https://doi.org/10.1016/j.cities.2018.09.021>

Delclós-Alió, X. y Miralles-Guasch, C. (2018). «Looking at Barcelona through Jane Jacobs's eyes: Mapping the basic conditions for urban vitality in a Mediterranean conurbation». Land Use Policy, 75, 505-517. $<$ https://doi.org/10.1016/j.landusepol.2018.04.026>

Downs, A. (2005). «Smart growth: Why we discuss it more than we do it». Journal of the American Planning Association, 71 (4), 367-378. <https://doi.org/10.1080/01944360508976707>

ForsyTH, A. (2015). "What is a walkable place? The walkability debate in urban design». Urban Design International, 20 (4), 274-292. <https://doi.org/10.1057/udi.2015.22>

FOSTER, M. S. (1981). From street car to superhighway. American city planners and urban transportation, 1900-1940. Philadelphia: Temple University Press.

Frank, L. D.; Sallis, J. F.; Saelens, B. E.; Leary, L.; Cain, K.; Conway, T. L. y Hess, P. M. (2010). "The development of a walkability index: application to the Neighborhood Quality of Life Study». British Journal of Sports Medicine, 44 (13), 924-933. <https://doi.org/10.1136/bjsm.2009.058701>

García, F. (2016). "Compacidad y densidad de las ciudades españolas». Eure, 42 (127), 5-27. <https://doi.org/10.4067/S0250-71612016000300001>

García-Palomares, J. C. (2010). «Urban sprawl and travel to work: the case of the metropolitan area of Madrid». Journal of Transport Geography, 18 (2), 197-213. <https://doi.org/10.1016/j.jtrangeo.2009.05.012>

GeHL, J. (2013). Cities for people. Washington, DC: Island Press.

GutiérReZ, A. y DOMĖNECH, A. (2018). «The mortgage crisis and evictions in Barcelona: identifying the determinants of the spatial clustering of foreclosures». European Planning Studies, 26 (10), 1939-1960. <https://doi.org/10.1080/09654313.2018.1509945>

Hajna, S.; Ross, N. A.; Brazeau, A. S.; Bélisle, P.; Joseph, L. y Dasgupta, K. (2015). «Associations between neighbourhood walkability and daily steps in adults: a systematic review and meta-analysis». BMC Public Health, 15 (1). <https://doi.org/10.1186/s12889-015-2082-x>

HiRT, S. (2016). "Rooting out mixed use: Revisiting the original rationales». Land Use Policy, 50, 134-147. <https://doi.org/10.1016/j.landusepol.2015.09.009>

HiRT, S. y ZAHM, D. (2012). The urban wisdom of Jane Jacobs. Nueva York: Routledge. IDESCAT, Institut d'Estadística de Catalunya (2017). Padró municipal d'habitants. Xifres oficials. Ambit metropolità. <https://www.idescat.cat/pub/?id=pmh\&n= 446\&geo=at:AT01>.

JACOBS, J. (1961). Muerte y vida de las grandes ciudades. Madrid: Capitán Swing.

Kent, J. L. y Thompson, S. (2014). "The Three Domains of Urban Planning for Health and Well-being». Journal of Planning Literature, 29 (3), 239-256. <https://doi.org/10.1177/0885412214520712> 
Knudsen, B.; Florida, R.; Stolarick, K. y Gates, G. (2008). «Density and creativity in U. S. Regions». Annals of the Association of American Geographers, 98 (2), 461-478. <https://doi.org/10.1080/00045600701851150>

KWAN, M. P. (2018). «The Limits of the Neighborhood Effect: Contextual Uncertainties in Geographic, Environmental Health, and Social Science Research». Annals of the American Association of Geographers, 108 (6), 1482-1490. <https://doi.org/10.1080/24694452.2018.1453777>

Maciejewska, M.; Miralles-Guasch, C. y Marquet, O. (2020). «Perfiles de población y uso de los transportes motorizados. Evidencias desde el campus de la Universitat Autònoma de Barcelona (UAB)». Documents d'Anàlisi Geogràfica, 66 (3), 629-648. $<$ https://doi.org/10.5565/rev/dag.553>

MarqueT, O.; HiPP, J. A. y Miralles-GuAsCH, C. (2017). «Neighborhood walkability and active ageing: A difference in differences assessment of active transportation over ten years». Journal of Transport \& Health, 7, 190-201. <https://doi.org/10.1016/j.jth.2017.09.006>

Marquet, O. y Miralles-Guasch, C. (2014). «Walking short distances. The socioeconomic drivers for the use of proximity in everyday mobility in Barcelona». Transportation Research Part A: Policy and Practice, 70, 210-222. <https://doi.org/10.1016/j.tra.2014.10.007>

- (2015). "The walkable city and the importance of the proximity environments for Barcelona's everyday mobility». Cities, 42, 258-266. <https://doi.org/10.1016/j.cities.2014.10.012>

Martín-Ramos, Á. (2012). "The Cerdà effect on city modernisation». Town Planning Review, 83 (6), 695-716. <https://doi.org/10.3828/tpr.2012.43>

Mateu, J.; Seguí, J. M. y Ruiz, M. (2017). «Mallorca y sus dinámicas metropolitanas: Proximidad y movilidad cotidiana en una isla-ciudad». Eure, 43 (129), 27-47. <https://doi.org/10.4067/S0250-71612017000200002>

MÉNDEZ, R. (2009). "Procesos recientes en regiones metropolitanas: transformaciones económicas y reorganización territorial. Algunas interpretaciones y debates actuales», 1-26.

Miralles-Guasch, C. (1998). «La movilidad de las mujeres en la ciudad. Un análisis desde la Ecología Urbana». Ecología Política, 15, 123-130.

- (2002). Ciudad y transporte: el binomio imperfecto. Barcelona: Ariel.

Miralles-Guasch, C. y Marquet, O. (2013). «Dinámicas de proximidad en ciudades multifuncionales». Ciudady Territorio: Estudios Territoriales, XLV (177), 503-512.

Miralles-Guasch, C.; Melo, M. M. y Marquet, O. (2015). «A gender analysis of everyday mobility in urban and rural territories: from challenges to sustainability». Gender, Place \& Culture, 23 (3), 398-417. <https://doi.org/10.1080/0966369X.2015.1013448>

Miralles-Guasch, C.; Vich, G. y Delclós-Alió, X. (2018). «La movilidad sostenible se juega en las escalas metropolitanas». En: BARON, N. y ROMERO, J. (eds.). Cultura territorial e innovación social ¿Hacia un nuevo modelo metropolitano en Europa del sur, 253-270. València: Universitat de València.

MontoyA, J. W. (2014). «Bogotá, urbanismo posmoderno y la transformación de la ciudad contemporánea». Revista de Geografía Norte Grande, 32 (57), 9-32. <https://doi.org/10.4067/S0718-34022014000100003> 
Morelli, V. G. y Salvati, L. (2010). Ad hoc urban sprawl in the Mediterranean city: Dispersing a compact tradition? Roma: Edizioni Nuova Cultura.

Muxí Martínez, Z.; Casanovas, R.; Ciocoletto, A.; Fonseca, M. y Gutiérrez VAldivia, B. (2011). "¿Qué aporta la perspectiva de género al urbanismo?». Feminismo/S, 105-129.

Nadai, M. de; Staiano, J.; Larcher, R.; Sebe, N.; Quercia, D. y Lepri, B. (2016). "The Death and Life of Great Italian Cities: A Mobile Phone Data Perspective». International World Wide Web Conference (IW3C2), 413-423. Montreal, Quebec, Canadá. <https://doi.org/10.1145/2872427.2883084>

OMS, Organización Mundial de la Salud (2010). «Recomendaciones mundiales sobre actividad física para la salud». Geneva: WHO Library Cataloguing-in-Publication (completo), 1-58. Recuperado de <https://www.who.int/dietphysicalactivity/publications/9789241599979/es/>.

Ortiz EsCalante, S. y GuTiÉRReZ Valdivia, B. (2015). «Planning from below: using feminist participatory methods to increase women's participation in urban planning». Gender and Development, 23 (1), 113-126. <https://doi.org/10.1080/13552074.2015.1014206>

Pallares-Barbera, M.; Badia, A. y Duch, J. (2011). "Cerdà and Barcelona: The need for a new city and service provision». Urbani Izziv, 22 (2), 122-136. <https://doi.org/10.5379/urbani-izziv-en-2011-22-02-005>

PaÜl I Agustí, D. (2017). «El Distrito 22@Barcelona como elemento de relocalización de las empresas de la ciudad. Un análisis de las antiguas y las nuevas ubicaciones de las sedes empresariales». Boletín de la Asociación de Geógrafos Españoles, 74, 185-204. <https://doi.org/10.21138/bage.2450>

SAlvati, L.; RonTOS, K. y Morelli, V. G. (2015). «Urban sprawl and implications for sustainable transportation: Analysing changing commuting patterns in a Mediterranean city region». International Journal of Sustainable Society, 7 (4), 333-352. <https://doi.org/10.1504/IJSSOC.2015.073553>

Salvati, L.; Sateriano, A. y Bajocco, S. (2013). "To grow or to sprawl? Land Cover Relationships in a Mediterranean City Region and implications for land use management». Cities, 30 (1), 113-121.

<https://doi.org/10.1016/j.cities.2012.01.007>

SCHUBERT, D. (2014). Contemporary Perspectives on Jane Jacobs: Reassessing the Impacts of an Urban Visionary. Hamburgo: Dirk Schubert.

Smith, M.; Hosking, J.; Woodward, A.; Witten, K.; MacMillan, A.; Field, A.; BAAS, P. y MACKIE, H. (2017). "Systematic literature review of built environment effects on physical activity and active transport - an update and new findings on health equity». International Journal of Behavioral Nutrition and Physical Activity, 14 (1), 1-27. <https://doi.org/10.1186/s12966-017-0613-9>

Southworth, M. (2005). "Designing the Walkable City». Journal of Urban Planning and Development, 131 (diciembre), 246. <https://doi.org/10.1061/(ASCE)0733-9488(2005)131:4(246)>

Sulis, P.; Manley, E.; Zhong, C. y BATTy, M. (2018). "Using mobility data as proxy for measuring urban vitality». Journal of Spatial Information Science, 16 (16), $137-162$. <https://doi.org/10.5311/JOSIS.2018.16.384> 
SunG, H.-G.; Go, D. H. y CHOI, C. G. (2013). «Evidence of Jacobs's street life in the great Seoul city: Identifying the association of physical environment with walking activity on streets». Cities, 35, 164-173. <https://doi.org/10.1016/j.cities.2013.07.010>

SunG, H.-G. y LeE, S. (2015). «Residential built environment and walking activity: Empirical evidence of Jane Jacobs' urban vitality». Transportation Research Part D: Transport and Environment, 41, 318-329. <https://doi.org/10.1016/j.trd.2015.09.009>

Sung, H.-G.; LeE, S. y CHEON, S. (2015). «Operationalizing Jane Jacobs’s Urban Design Theory: Empirical Verification from the Great City of Seoul, Korea». Journal of Planning Education and Research, 35 (2), 117-130. <https://doi.org/10.1177/0739456X14568021>

VAlenzuela-Montes, L. M. y TAlaVERA-García, R. (2015). «Entornos de movilidad peatonal: Una revisión de enfoques, factores y condicionantes». Eure, 41 (123), 5-27. <https://doi.org/10.4067/S0250-71612015000300001>

Whyte, W. H. (1980). The Social Life of Small Urban Spaces. Washington, DC: Conservation Foundation.

<https://doi.org/10.1177/089124168201000411> 